\title{
Immunologic Factors in Human Milk and Disease Prevention in the Preterm Infant
}

\author{
Katherine E. Gregory • W. Allan Walker
}

Published online: 26 September 2013

(c) Springer Science + Business Media New York 2013

\begin{abstract}
Exposure to the immunologic factors found in maternal breast milk has been shown to compensate for the immature immune system that characterizes the preterm infant. Feeding preterm infants with human milk not only provides protection from disease pathogenesis and ensures an optimal source of nutrients in the short term, but it also helps to ensure the development of a healthy immune response in the long term. Here, we review the immunological significance and antiinflammatory effects of a selection of the bioactive molecules found in human milk and how they may differ under conditions of preterm birth. In addition, the role that human milk plays in the intestinal colonization of the preterm infant and why this is important to short- and long-term health outcomes are discussed.
\end{abstract}

Keywords Breast milk - Preterm infant - Immunology · Intestinal colonization $\cdot$ Cytokines $\cdot$ Antibodies

K. E. Gregory ( $\square)$

W.F. Connell School of Nursing, Boston College, 140 Commonwealth Avenue, Chestnut Hill, MA 02467, USA

e-mail: katherine.gregory.2@bc.edu

K. E. Gregory

Brigham and Women's Hospital, Boston, MA, USA

W. A. Walker

Mucosal Immunology and Biology Research Center,

Massachusetts General Hospital for Children, Harvard Medical

School, 114 16th Street, Charlestown, MA, USA

e-mail: wwalker@partners.org

\section{Introduction}

Human milk has been recommended as the optimal and exclusive source of early nutrition for all infants following birth and until at least 6 months of age [1,2]. Human milk is the ideal source of early infant nutrition as a result of potent immune factors and a unique composition of nutrients that evolve in tandem with the growth and developmental needs of the infant. For infants who are born preterm, exposure to the immune factors of human milk is of paramount importance as these infants are born with an immature immune system. For these infants, human milk not only provides protection from disease pathogenesis in the short term, but it also helps to ensure the development of a healthy immune response in the long term. In this article, we will provide an overview of what is known about the immunological profile of preterm infants and review in depth the immunological factors found in human milk that are important for disease prevention and health.

\section{Immunological Profile of the Preterm Infant}

Preterm birth (PTB), defined as birth occurring prior to 37 weeks of gestation [3], persists as a leading perinatal health problem facing women and children. The causes are not fully understood, and preventative measures are lacking [4-6]. The PTB rate is currently reported at $12.8 \%$ of all births in the USA [7, 8] and affects as many as 15 million infants worldwide [9]. The implications of PTB on human health have been well documented [10-17]. One of the leading causes of morbidity and mortality among infants born preterm is infection (bacteremia, pneumonia, meningitis) during the early neonatal period, occurring approximately in one in six infants. A disproportionate number of 
fatal infections occur in the smallest and most premature infants [18, 19] who require invasive interventions disrupting the protective mucosal and epithelial barriers in the face of inadequate innate immunity [20].

Innate Immunity in the Preterm Infant

Innate immunity is of primary importance during early life while a fully functional adaptive immune system is developing through interactions with the environment at the mucosal surfaces. The innate immune system specific to the preterm infant is characterized by developmental immaturities that include deficiencies in the number and function of white blood cells, reduced pattern-recognition receptors (PRR) such as Toll-like receptors (TLRs), and limited tight junction (TJ) function of defensins such as Paneth cells $[20,21]$. These characteristics common to the preterm infant result in an inadequate extracellular elimination of bacterial infections and aberrant recruitment of immune cells to the site of infection or inflammation [22]. Factors such as TLR4, MD-2, a molecule that participates in the LPS response on TLR4, and other components of the innate immune system are found in human milk and, in the case of the preterm infant, may provide an innate immune response that would not otherwise exist.

Infants who are born preterm start life at an immunological disadvantage when compared to their full-term counterparts, who receive maternal antibodies that are transferred via the placenta during the later stages of pregnancy [23, 24]. Preterm infants are also prone to aberrant intestinal colonization as a result of routine use of antibiotics at birth, often limited exposure to breast milk, increased incidence of birth by Cesarean section, and prolonged hospitalization during early infancy. These factors place infants at a microbial disadvantage and high-risk for inflammatory immune diseases [25, 26], both in the early neonatal period and later in life. Exposure to the immunological factors found in maternal breast milk has been shown to contribute to the development of a more optimal immune response in the preterm infant, thereby overcoming some of the deficiencies of the innate immune function.

\section{Immunological Factors Found in Human Milk}

Breast milk has been called the gold standard for protective nutrients [27] as a result of the potent immunological properties of this dynamic body fluid. As outlined in Table 1, exposures to the protective immunological factors found in human milk counteract the deficiencies that characterize the preterm infant's innate immune system and contribute to the development of the overall immune function. Here, we review the immunological significance and effects of a selection of the bioactive molecules found in human milk under conditions of premature birth. In addition, the role that human milk plays in the intestinal colonization of the preterm infant is also discussed.

Immunoglobulins

Immunoglobulin-producing B cells are not fully functional at birth, which causes a delayed activation of B cells and, in turn, delayed maturation of antibody-producing plasma cells. These delays result in a temporary deficit of secretory $\operatorname{IgA}$ (SIgA) production. This deficit is especially problematic for the preterm infant because SIgA is not absorbed by the neonatal gut, allowing these antibodies to protect the infant's intestinal mucosa from potentially harmful pathogens. Thus, the absence of SIgA leaves the preterm intestinal mucosa highly vulnerable to pathogenic microorganisms.

SIgA has been measured and compared in the colostrum and mature milk of women who deliver preterm and or at term. These studies have shown that there is significantly more SIgA in the breast milk of women who deliver preterm, with the highest concentrations in the colostrum of women who deliver early $[28 \bullet, 29,30]$. SIgA concentrations have been shown to taper over time. This taper is slower and more gradual in the milk of women who deliver preterm when compared to that of women who deliver at term [31]. It is speculated that an immunological adaptive response may be present in the breast milk of women who deliver preterm, providing protection to the preterm intestinal mucosa from microorganisms.

IgG is of importance to the preterm infant for its opsonization and agglutination mechanism in pathogen

Table 1 Protective immunologic factors found in human milk mitigate characteristics of immature innate immunity

\begin{tabular}{|l|l|}
\hline Characteristics of Innate Immunity in the Preterm Infant & Protective Immunologic Factors Found in Human Milk \\
\hline $\begin{array}{l}\text { Lack of maternal antibodies transferred via the placenta during } \\
\text { late pregnancy }\end{array}$ & $\begin{array}{l}\text { Immunoglobulins: } \\
\text { Secretory IgA, IgG }\end{array}$ \\
\hline Inadequate extracellular elimination of bacterial infections & $\begin{array}{l}\text { Cytokines: } \\
\text { IL-6, IL-8, TNF- } \alpha, \text { TGF } \beta 1 \text { and TGF } \beta 2\end{array}$ \\
\hline $\begin{array}{l}\text { Reduced pattern-recognition receptor (PRR) and tight junction } \\
\text { (TJ) function contributing to inappropriate inflammation }\end{array}$ & $\begin{array}{l}\text { Growth Factors: } \\
\text { EGF, TGF- } \alpha \text { and TGF- } \beta\end{array}$ \\
\hline Aberrant intestinal colonization & $\begin{array}{l}\text { Microbiologic Factors: } \\
\text { Lactoferrin, Human Milk Oligosaccharides, Probiotic Bacteria }\end{array}$ \\
\hline
\end{tabular}


protection. IgG is transferred from the mother to the fetus via the placenta during the later stages of pregnancy, thereby providing immune protection to the fetus and the neonate. Infants who are born preterm do not receive the full benefit of this immune protection and are IgG deficient at birth when compared to their fullterm counterparts. IgG, much like SIgA, while found in small concentrations in breast milk, is of great importance to the preterm infant. Past studies have shown that $\operatorname{IgG}$ is detected in the majority of colostrum samples and that the concentrations of this antibody are similar in the colostrum of women who deliver preterm and at term [32-34]. However, more recent studies suggest that $\operatorname{IgG}$ is higher in the colostrum obtained from women who give birth prior to 32 weeks gestation [31]. Evidence suggests that an $\mathrm{IgG} F c$ receptor is expressed on the luminal surface of the enterocytes in the fetal human intestine [35]. These receptors may function after birth as a transport system for $\mathrm{IgG}$, facilitating the uptake of $\operatorname{IgG}$ in breast milk, contributing to the immune response of the premature infant. These findings reinforce the speculation that there may be a unique adaptive response mechanism associated with the lactation pattern of women who deliver preterm.

The neonatal immune system is capable of producing IgM in response to pathogens. Thus, it is not surprising that the IgM concentration in colostrum is low, and detected in approximately $20 \%$ of samples [31]. In studies that have evaluated IgM in the milk of women who deliver at different points in gestation, no significant differences have been observed in the concentrations of IgM in the milk [31, $33,34,36]$.

\section{Cytokines}

Breast milk functions to influence the immunological development of the infant through the action of cytokines, one of the most important bioactive components found in human milk. Cytokines are pluripotent polypeptides that act as chemical messengers among cells. These proteins operate in a complex stepwise approach, producing a cascade of effects that contribute to the development and function of the immune system. Cytokines regulate the inflammatory responses stimulated by an antigenic challenge. Through the use of immunoassays, several cytokines have been measured in human milk [28•, 37, 38, 39•]. The majority of research conducted on cytokines in human milk has been done using milk obtained from women who gave birth to full-term infants. However, measurements of milk from women who gave birth to preterm infants show important differences in the cytokine profile that vary with length of gestation.
IL-6 is a cytokine with both pro- and antiinflammatory functions important to the immune system. IL-6 has been highly correlated with IgA, TGF- $\beta 1$, and TGF$\beta 2$ in the milk from women following PTB. These findings support the hypothesis that IL-6 plays a significant role in the development and differentiation of IgA-producing cells [37, 40]. When measured in colostrum, transitional milk, and mature milk obtained from women who deliver at term or prematurely, IL-6 has been shown to be highest in the colostrum, tapering off as the milk matures [28•, 41]. Interestingly, when cytokines were measured in the milk obtained from women after birth at different points of gestation, the highest concentrations of IL- 6 were observed between 30 weeks and term, and then lowest concentrations prior to 30 weeks of gestation. Slightly different results have been reported from a study that compared IL- 6 in the milk of women who deliver very preterm (24-31 weeks) with that of women who deliver at term (38-41 weeks). IL-6 was found to be higher in the transitional breast milk of women in the very preterm group when compared to the term group; however, this difference decreased in mature milk, suggesting that cytokine content of breast milk decreases with ongoing lactation [39•]. In addition, although IL-6 was higher in the preterm group, multiple regression analysis conducted in this study indicated that the difference was not associated with gestational age at birth, but cesarean delivery. Other discrepancies among studies may be explained by how colostrum, transitional, and mature milk are defined, as well as how infants are classified based on their gestational age at birth.

Overall, the findings relative to IL-6 suggest that human milk may have the ability to compensate for the immunological immaturity common to premature infants, but perhaps only after a certain point in gestation. Thus, infants born extremely preterm (i.e., before 30 weeks) appear to be at an even greater immunological disadvantage; not only is their immature immune system ill prepared for the extrauterine environment, but also the compensatory properties found in human milk that support development of the immune system and protection from infection are less abundant.

IL-8 is typically secreted by macrophages, cells with TLRs that are involved in the innate immune response. For preterm infants, who have a delayed innate immune system that is slow to respond to antigens, exposure to IL-8 in human milk is of great importance. IL- 8 has consistently been shown to decrease with duration of lactation, regardless of gestational age. The results are inconsistent when compared across gestational ages. Ustundag and colleagues found no differences in the levels of IL-8 based on gestation [41], while Castellote and colleagues, as well 
as Mehta and Petrova, observed a lower amount of IL-8 measured in the colostrum from women who delivered prior to 30 weeks as compared to the colostrum obtained at later gestational ages [28•, 39•]. The decrease in IL-8 over the duration of lactation underscores the bioactive nature of human milk, which adjusts to the developing competence of the human immune system.

Similar to IL-6 and IL-8, IL-10 and TNF- $\alpha$ have been shown to vary based on gestational age at birth and duration of lactation. While the measurements of these cytokines are far lower when compared to IL-6 and IL-8, concentrations of IL-10 and TNF- $\alpha$ are highest in the milk of women who give birth between 30 and 37 weeks of gestation and lowest in women who give birth prior to 30 weeks of gestation [28•]. The high concentration of cytokines in colostrum when compared to more mature milk emphasizes the importance of early exposure to human milk as an essential component of the developing immune system during infancy, especially in the preterm infant.

\section{Growth Factors}

Growth factors are among the important bioactive components found in human milk. Of particular importance to the preterm infant are epidermal growth factor (EGF) and transforming growth factor alpha and beta (TGF- $\alpha$ and TGF- $\beta$ ). These specific growth factors promote the functional development of the gastrointestinal mucosa beginning at birth, and when working together with antiinflammatory IL-10, they allow the immune system to tolerate antigens from nutritional exposures and specific microbiota found in the gut. In addition, these growth factors participate in the immune system to downregulate the inflammatory response and promote repair of damaged intestinal cells. The healing aspect of growth factors is of particular importance to preterm infants who may suffer intestinal injury including necrotizing enterocolitis (NEC).

Not unlike several other bioactive immune factors important to the preterm infant, EGF and TGF- $\alpha$ have been measured at higher concentrations in the milk of women who deliver preterm when compared to the milk of women who deliver at term [28•, 42]. In one study, where total protein concentrations did not vary based on gestational age at birth or in the first 7 days of lactation, both EGF and TGF- $\alpha$ were significantly higher during the first post-natal week in the milk of women who delivered very preterm (prior to 27 weeks of gestation) when compared with women who delivered between 32 and 36 weeks of gestation or between 38 and 42 weeks of gestation [42]. Furthermore, this study showed that both of these growth factors were measured at the highest concentrations in the first week following birth and then tapered off gradually during the early neonatal period, but remained highest in the very preterm group even as the number of days of lactation increased. A more recent study with a similar design also showed that the EGF concentration in the milk of women who deliver before 30 weeks of gestation is higher than that of women who deliver later in pregnancy [28•]. The concentrations of TGF- $\beta$ measured in this study were highest in the milk of women who delivered preterm (between 30 and 37 weeks), not in the very preterm group, who delivered before 30 weeks. The concentrations of TGF- $\beta 1$ gradually decreased over time in all groups, with the highest concentrations measured in colostrum and the lowest concentrations measured in mature milk. This was slightly different for TGF- $\beta 2$, where concentrations tapered less significantly from colostrum to mature milk and were slightly lower in the colostrum of the women who delivered very preterm [28•].

The high concentration of growth factors such as EGF measured in human milk, especially the milk of women who deliver preterm, very likely contributes to the protective effect of human milk for neonatal gastrointestinal diseases such as NEC. The unique importance of EGF to the immature gut is further supported by the fact that EGF receptors are found in both the fetal and neonatal intestine [43]. Finally, the inverse relationship between EGF concentrations and gestational age support the speculation that growth factor content in human milk may be a maternal adaptation to premature delivery.

\section{Lactoferrin}

Lactoferrin, a prominent whey protein found in breast milk, is not only a prebiotic, but also antiinfective. Lactoferrin has been shown to reduce the production of inflammatory cytokines in monocytes and, in the presence of a probiotic (Lactobacillus GG), has enhanced defenses against infective enteric pathogens such as $E$. coli in the small intestines [44]. In studies evaluating bioactive compounds in the breast milk of women who deliver preterm, lactoferrin has been shown to decrease over time, with higher levels observed during early lactation and lower levels observed by the end of the first month of lactation [38•]. When compared to the breast milk of women who deliver at term, lactoferrin has been measured at lower concentrations in the milk of women who deliver preterm [38•]. The higher concentration of lactoferrin in the milk of women who deliver at term might be explained by the need for higher concentrations of this protein in more mature infants, which serves as a growth factor for Bifidobacterium and Lactobacillus species. 


\section{Microbiology of Human Milk and Intestinal Colonization of the Preterm Infant}

The implementation of advanced molecular approaches in the study of human microbiology and discoveries generated by the Human Microbiome Project have resulted in a renewed emphasis on the importance of microbial health [45-49]. While complex microbial-host relationships are understood to play a role in health and disease across the lifespan, never are they more important than during infancy, when the intestinal microbiome is first assembled [50]. This assembly process is highly dependent on nutritional exposures during early infancy, where breast milk is likely to play a leading role as a result of the diversity of microbes that are present in this body fluid and due to the molecular mechanisms that are triggered by human milk oligosaccharides (HMO) found in breast milk, which act as a source of energy for desirable bacteria that are important to the establishment of an optimal microbiome.

The most recent metagenomic studies employing Illumina sequencing have shown that there is a highly diverse community of bacteria present in human milk samples [51•]. The population of bacteria found in the human milk samples included in this study was primarily aligned with the phyla of Proteobacteria (65\%) and Firmicutes (34\%) and the genera of Pseudomonas (61.1\%), Staphylococcus (34.4\%), and Streptococcus (0.5\%). Within these overall categories, 177 unique bacterial genera were measured in human milk samples. In another study involving $16 \mathrm{~S}$ rRNA sequencing, stool samples obtained from breastfed infants were compared to those of formula-fed infants [52•]. In this study, breastfed infants had a higher proportion of Bacteroidetes and a lower proportion of Firmicutes and Verrucomicrobia when compared to formula-fed infants [52•]. The diversity of the bacterial communities found within human milk likely contributes to the overall intestinal colonization that occurs during infancy, but the exact mechanisms are not fully understood. The differences observed in this study may be explained by the microbial diversity found within the milk itself or by the presence of HMO within human milk (and lacking in infant formula) and, in turn, the intestinal microbiome of the breastfed infant who is colonized with health-promoting bacteria that are capable of metabolizing HMO.

Regardless of the mechanisms, studies using both Illumina and 16S rRNA sequencing have shown that the human milk microbiome, not unlike the microbiome found in other bodily habitats, is highly unique in its composition to each individual. The unique nature of the microbiome found in human milk is very likely influenced by the intestinal microbiome of the mother [53]. It has been hypothesized that during the perinatal period, the permeability of the maternal gut allows for uptake of bacteria into the mother's blood stream and then into the breast milk. Thus, the microbial population of the maternal gut has a potentially strong influence on the microbes found in breast milk and very likely contributes to the symbiotic effect of HMO on breast milk microbiota, which is ultimately an important part of infant nutrition and early intestinal colonization. In term infants, this process stimulates growth of Bifidobacteria, Lactobacillus, and Bacteroides, all of which have a protective effect on the newborn intestine. Unfortunately, the preterm infant does not benefit from this pattern of colonization, leaving the immature gut especially vulnerable [54]. Further study of the microbiome characterizing human milk investigating the influence of the maternal gestation (i.e., term vs. preterm milk) and Holder pasteurization $\left(65^{\circ} \mathrm{C}\right.$ for $\left.5-30 \mathrm{~min}\right)$ commonly used by milk banks. This will enhance our understanding of the unique properties of human milk and the role that it plays in the intestinal colonization of the preterm infant.

\section{Conclusion: Human Milk Exposure and Disease Prevention in the Preterm Infant}

Children born preterm are characterized by an immature immune response and, as a result, are especially vulnerable to infectious and immune-mediated disease. As outlined in this article, human milk provides a unique composition of bioactive factors that have the ability to overcome the immature immune response and thus may prevent inflammatory diseases such as NEC in the short term, resulting in optimal growth and neurodevelopment in the long term.

NEC remains the most catastrophic gastrointestinal disease among preterm infants. The mechanisms underpinning NEC are multifactorial and remain poorly understood. The only consistently identified protective strategy against NEC is exposure to human milk [26, 55]. Preterm infants who are breast fed have been shown to be at a sixfold to tenfold lower risk of NEC when compared to formula-fed infants [56]. The protective mechanisms provided by human milk against this disease are likely associated with optimal intestinal colonization, which helps to prevent against inflammation and infection by pathogenic bacteria. In addition, preterm infants fed with human milk have the advantage of an innate immune system that is programmed by the powerful immunological factors found in human milk. As a result, these children are better equipped to mount an appropriate inflammatory response that may prevent the disease pathogenesis common to NEC. In sum, providing human milk to preterm infants is of paramount importance to enhancing both short- and long-term health outcomes as a result of the unique immunologic properties found in this optimal source of infant nutrition. 


\section{Compliance with Ethics Guidelines}

Conflict of Interest Katherine E. Gregory declares that she has no conflict of interest. W. Allen Walker has received gifts from Mead Johnson Research and had travel/accommodation expenses covered or reiumbursed from Mead Johnson Research and Dannon Infant Nutrition.

Human and Animal Rights and Informed Consent This article does not contain any studies with human or animal subjects performed by any of the authors.

\section{References}

Articles of particular interest, published recently, have been highlighted as:

- Of importance

1. Section on Breastfeeding. Breastfeeding and the use of human milk. Pediatrics. 2012;29(3):e827-41.

2. World Health Organization. Global strategy for infant and young child feeding. Geneva: Switzerland; 2001.

3. Beck S, Wojdyla D, Say L, et al. The worldwide incidence of preterm birth: a systematic review of maternal mortality and morbidity. Bull World Health Organ. 2010;88(1):31-8.

4. Steer PJ. The epidemiology of preterm labour-why have advances not equated to reduced incidence? BJOG. 2006;113: $1-3$.

5. Institute of Medicine. Preterm Birth: Causes, Consequences, and Prevention. In: Behrman RE, Stith Butler A, editors. Washington DC: National Academies Press (US); 2007.

6. ACOG Practice Bulletin. Assessment of risk factors for preterm birth. Clinical management guidelines for obstetrician-gynecologists. Number 31, October 2001. (Replaces Technical Bulletin number 206, June 1995; Committee Opinion number 172, May 1996; Committee Opinion number 187, September 1997; Committee Opinion number 198, February 1998; and Committee Opinion number 251, January 2001). Obstet Gynecol. 2001; 98(4):709-16.

7. Martin JA, Hamilton BE, Sutton PD, et al. Births: final data for 2005. National vital statistics reports: from the Centers for Disease Control and Prevention, National Center for Health Statistics, National Vital Statistics System 2007;56(6):1-103.

8. Ashton DM, Lawrence HC, Adams NL, Fleischman AR. Surgeon General's Conference on the Prevention of Preterm Birth. Obstet Gynecol. 2009;113(4):925-30.

9. Born Too Soon: The Global Action Report on Preterm Birth. Geneva: March of Dimes, Save the Children, and WHO, 2012.

10. Eichenwald EC, Stark AR. Management and outcomes of very low birth weight. N Engl J Med. 2008;358(16):1700-11.

11. Mura T, Picaud JC, Larroque B, et al. Cognitive impairment at age 5 years in very preterm infants born following premature rupture of membranes. J Pediatr. 2013;. doi:10.1016/j.jpeds.2013. 01.039 .

12. Saigal S, Doyle LW. An overview of mortality and sequelae of preterm birth from infancy to adulthood. Lancet. 2008;371(9608): 261-9.

13. Petrou S, Sach T, Davidson L. The long-term costs of preterm birth and low birth weight: results of a systematic review. Child Care Health Dev. 2001;27(2):97-115.
14. Russell RB, Green NS, Steiner CA, et al. Cost of hospitalization for preterm and low birth weight infants in the United States. Pediatrics. 2007;120(1):e1-9.

15. Marlow N, Wolke D, Bracewell MA, Samara M. Neurologic and developmental disability at six years of age after extremely preterm birth. N Engl J Med. 2005;352(1):9-19.

16. Goldenberg RL, Culhane JF, Iams JD, Romero R. Epidemiology and causes of preterm birth. Lancet. 2008;371(9606):75-84.

17. Moster D, Lie RT, Markestad T. Long-term medical and social cosequences of preterm birth. N Engl J Med. 2008;359(3): 262-73.

18. Stoll BJ, Hansen N, Fanaroff AA, et al. Late-onset sepsis in very low birth weight neonates: the experience of the NICHD Neonatal Research Network. Pediatrics. 2002;110:285-91.

19. Stoll BJ, Hansen N. Infections in VLBW infants: studies from the NICHD Neonatal Research Network. Semin Perinatol. 2003; 27(4):293-301.

20. Sharma AA, Jen R, Butler A, Lavoie PM. The developing human preterm neonatal immune system: a case for more research in this area. Clin Immunol. 2012;145(1):61-8.

21. Levy O. Innate immunity of the newborn: basic mechanisms and clinical correlates. Nat Rev Immunol. 2007;7(5):379-90.

22. Nussbaum C, Sperandio M. Innate immune cell recruitment in the fetus and neonate. J Reprod Immunol. 2011;90(1):74-81.

23. van den Berg JP, Westerbeek EA, van der Klis FR, et al. Transplacental transport of IgG antibodies to preterm infants: a review of the literature. Early Hum Dev. 2011;87(2):67-72.

24. Palmeira P, Quinello C, Silveira-Lessa AL, et al. IgG placental transfer in healthy and pathological pregnancies. Clin Dev Immunol. 2012;. doi:10.1155/2012/985646.

25. Newburg DS, Walker WA. Protection of the neonate by the innate immune system of developing gut and of human milk. Pediatr Res. 2007;61(1):2-8.

26. Torrazza RM, Neu J. The developing intestinal microbiome and its relationship to health and disease in the neonate. J Perinatol. 2011;31(S1):S29-34.

27. Walker A. Breast milk as the gold standard for protective nutrients. J Pediatr. 2010;156(2):S3-7.

28. - Castellote C, Casillas R, Ramírez-Santana C, Pérez-Cano FJ, Castell M, Moretones MG, et al. Premature delivery influences the immunological composition of colostrum and transitional and mature human milk. J Nutr 2011;141(6):1181-7. The immune factors present in breastmilk vary based on the duration of gestation as well as lactation. The maternal lactogeneic compensatory mechanisms that are thought to help protect preterm infants appear to function after 30 weeks of gestation, leaving most preterm infants especially vulnerable.

29. Araujo ED, Goncalves AK, Cornetta Mda C, et al. Evaluation of the secretory immunoglobulin A levels in the colostrum and milk of mothers of term and pre-term newborns. Braz $\mathrm{J}$ Infect Dis. 2005;9(5):357-62.

30. Ballabio C, Bertino E, Coscia A, et al. Immunoglobulin-A profile in breast milk from mothers delivering full term and preterm infants. Int J Immunopathol Pharmacol. 2007;20(1):119-28.

31. Koenig A, de Albuquerque Diniz EM, Barbosa SF, Vaz FA. Immunologic factors in human milk: the effects of gestational age and pasteurization. J Hum Lact. 2005;21(4):439-43.

32. Gross S, Buckley RH, Wakil SS. Elevated IgA concentration in milk produced by mothers delivered of preterm infants. J Pediatr. 1981;1981(99):389-93.

33. Suzuki S, Lucas A, Lucas PJ, Coombs RR. Immunoglobulin concentrations and bacterial antibody titres in breast milk from mothers of 'preterm' and 'term' infants. Acta Paediatrica Scandinavica. 1983;72(5):671-7. 
34. Mathur NB, Dwarkadas AM, Sharma VK, et al. Anti-infective factors in preterm human colostrum. Acta Paediatrica Scandinavica. 1990;79(11):1039-44.

35. Shah U, Dickinson BL, Blumberg RS, et al. Distribution of the IgG Fc receptor, FcRn, in the human fetal intestine. Pediatr Res. 2003;53(2):295-301.

36. Gross SJ, Buckley RH, Wakil SS, McAllister DC, David RJ, Faix RG. Elevated IgA concentration in milk produced by mothers delivered of preterm infants. J Pediatr. 1981;99(3):389-93.

37. Garofalo R. Cytokines in human milk. J Pediatr. 2010;156:S36-40.

38. - Mehta R, Petrova A. Biologically active breast milk proteins in association with very preterm delivery and stage of lactation. J Perinatol. 2011;31(1):58-62. Breast milk from women who deliver preterm is a better source of bioactive factors that promote antibacterial and anti-inflammatory mechanisms. However, it has also been shown to be deficient in leptin, which is imporant to neuroendocrine function.

39. - Mehta R, Petrova A. Very preterm gestation and breastmilk cytokine content during the first month of lactation. Breastfeed Med. 2011;6(1):21-4. Contrary to other reports, delivery following extremely preterm gestation does not significantly influence the inflammatory cytokine content of breasmilk during the first month of lactation. This is unfortunate, given the immature innate and adaptive immunity that characterizes the preterm infant.

40. Brandtzaeg P. Nature and function of gastrointestinal antigenpresenting cells. Allergy. 2001;56:16-20.

41. Ustundag B, Yilmaz E, Dogan Y, et al. Levels of cytokines (IL1beta, IL-2, IL-6, IL-8, TNF-alpha) and trace elements ( $\mathrm{Zn}, \mathrm{Cu})$ in breast milk from mothers of preterm and term infants. Mediators Inflamm. 2005;2005(6):331-6.

42. Dvorak B, Fituch CC, Williams CS, Hurst NM, Schanler RJ. Increased epidermal growth factor levels in human milk of mothers with extremely premature infants. Pediatr Res. 2003;54(1):15-9.

43. Chailler P, Menard D. Ontogeny of EGF receptors in the human gut. Front Biosci. 1999;4:D87-101.

44. Sherman MP, Bennett SH, Hwang FF, Yu C. Neonatal small bowel epithelia: enhancing anti-bacterial defense with lactoferrin and lactobacillus GG. Biometals. 2004;17(3):285-9.

45. Devaraj S, Hemarajata P, Versalovic J. The human gut microbiome and body metabolism: implications for obesity and diabetes. Clin Chem. 2013;. doi:10.1373/clinchem.2012.187617.
46. Human Microbiome Project Consortium. Structure, function and diversity of the healthy human microbiome. Nature. 2012;486 (7402):207-14.

47. Human Microbiome Project Consortium. A framework for human microbiome research. Nature. 2012;486(7402):215-21.

48. Turnbaugh P, Ley R, Hamady M, et al. The human microbiome project. Nature. 2007;449:804-10.

49. Turnbaugh P, Ley R, Mahowald M, et al. An obesity-associated gut microbiome with increased capacity for energy harvest. Nature. 2006;444:1027-31.

50. Vael C, Desager K. The importance of the development of the intestinal microbiota in infancy. Curr Opin Pediatr. 2009;21 (6):794-800.

51. - Ward TL, Hosid S, Ioshikhes I, Altosaar I. Human milk metagenome: a functional capacity analysis. BMC Microbiol. 2013;13(1):116. Using advanced sequencing technology and pooled milk samples from ten donors, the bacterial diveristy of human milk was measured. In addition, the human milk metagenome was compared between breast- and formula-fed infants, revealing that there are numerous differences between the human milk metagenome and the infant fecal metagenome in both breast- and formula-fed infants.

52. - Donovan SM, Wang M, Li M, et al. Host-microbe interactions in the neonatal intestine: role of human milk oligosaccharides. Adv Nutr. 2012;3(3):450S-5S. The abundance and diversity of HMO found in breast milk and their extensive physiological actions may very well explain the differences in the microbiota observed in breast- and formula-fed infants.

53. Collado MC, Laitinen K, Salminen S, Isolauri E. Maternal weight and excessive weight gain during pregnancy modify the immunomodulatory potential of breast milk. Pediatr Res. 2012;72 (1):77-85.

54. Mshvildadze M, Neu J. The infant intestinal microbiome: friend or foe? Early Hum Dev. 2010;86(Suppl 1):67-71.

55. Neu J, Walker WA. Necrotizing enterocolitis. N Engl J Med. 2011;364(3):255-64.

56. Sisk PM, Lovelady CA, Dillard RG, Gruber KJ, O'Shea TM. Early human milk feeding is associated with a lower risk of necrotizing enterocolitis in very low birth weight infants. J Perinatol. 2007;27(7):428-33. 